Classification

Physics Abstracts

$61.80 \mathrm{~J}-71.55$

\title{
Etude des centres à électrons créés par irradiation de protons dans InP:n
}

\author{
S. Loualiche (*), P. Rojo, G. Guillot et A. Nouailhat \\ Laboratoire de Physique de la Matière (LA 358), Institut National des Sciences Appliquées de Lyon, \\ 20, avenue Albert Einstein, 69621 Villeurbanne Cedex, France
}

(Reçu le 25 juillet 1983, révisé le 21 novembre, accepté le 8 décembre 1983)

\begin{abstract}
Résumé. - Les expériences ont été faites sur du matériau LEC non intentionnellement dopé. Les irradiations, avec des protons de $100 \mathrm{keV}$, sont effectuées aux températures $6 \mathrm{~K}, 77 \mathrm{~K}, 300 \mathrm{~K}$ et les pièges créés étudiés par méthodes capacitives : concentrations, profils et domaines de stabilités ont été déterminés. Les irradiations à $6 \mathrm{~K}$ et $77 \mathrm{~K}$ donnent les mêmes résultats expérimentaux. Quatre pièges principaux sont créés, identiques à ceux observés après irradiation aux électrons dans des conditions équivalentes. Il existe, de $77 \mathrm{~K}$ à $300 \mathrm{~K}$, deux stades de recuit à $100 \mathrm{~K}$ et $160 \mathrm{~K}$. L'un des centres au moins (A.3) provient d'un processus complexe mettant en jeu une diffusion avant stabilisation. Une irradiation à $300 \mathrm{~K}$ est équivalente à une irradiation à $77 \mathrm{~K}$ suivie d'un recuit à $300 \mathrm{~K}$.
\end{abstract}

\begin{abstract}
Schottky diodes made on $\mathrm{n}$ type LEC InP samples have been irradiated by $100 \mathrm{keV} \mathrm{H}^{+}$at $6 \mathrm{~K}$, $77 \mathrm{~K}$ and $300 \mathrm{~K}$ and the created electron traps studied by transient capacitance techniques : concentration, profiles and annealing temperature ranges have been determined. Irradiations at $6 \mathrm{~K}$ and $77 \mathrm{~K}$ give the same results : between $77 \mathrm{~K}$ and $300 \mathrm{~K}$, four electron traps have been detected, identical to those observed after electron irradiation at $20 \mathrm{~K}$. The two shallowest disappear after annealing at $100 \mathrm{~K}$ and $160 \mathrm{~K}$ respectively. One observed trap at least comes from a diffusion process before stabilization. Irradiation at room temperature gives similar results to an irradiation at $77 \mathrm{~K}$ followed by $300 \mathrm{~K}$ annealing.
\end{abstract}

\section{Introduction.}

L'implantation ionique dans InP produit des effets de compensation $[1,2]$. Ceux-ci peuvent être mis à profit pour fabriquer des zones semi-isolantes ou, au contraire, éliminés pour optimiser l'activité électrique des ions implantés. Il apparaît donc particulièrement important de connaître les propriétés des défauts créés et, dans un premier temps, ceux créés par des protons. Ceux-ci, utilisés à dose réduite $\left(<10^{11} \mathrm{~cm}^{-2}\right)$, créent les mêmes types de défaut que les électrons, la différence étant que les protons donnent naissance à des centres localisés dans le solide, autour de la profondeur d'arrêt $\left(R_{\mathrm{p}}\right)$ alors que les électrons de $1 \mathrm{MeV}$ donnent des centres uniformément répartis dans l'échantillon. Cette propriété importante des protons permet de vérifier :

i) si les centres créés sont des défauts qui diffusent ou des défauts qui restent sur place;

ii) si un défaut qui naît après un recuit résulte de la transformation sur place d'un défaut initial ou d'une

(*) Adresse actuelle C.N.E.T. (ICM), Route de Trégastel, 22301 Lannion, France. migration à longue distance plus stabilisation donnant naissance à un complexe et ainsi comme dans le cas de l'étude des recuits des défauts d'irradiation dans $\mathrm{GaAs}$, de remonter aux mécanismes de transformation ou d'annihilation des pièges créés par l'irradiation [3].

Cette méthode est donc bien adaptée à l'étude des défauts d'irradiation dans InP car différents travaux [4-6] ont montré, d'une part, que les taux d'introduction faibles des défauts créés dans InP de type $n$ semblent en faveur de défauts observés de type secondaire et, d'autre part, que certains d'entre eux diffusent à basse température.

\section{Conditions expérimentales.}

Le matériau utilisé est de l'InP type $\mathrm{n}$ massif LEC de Metal Research orienté $\langle 100\rangle$ non intentionnellement dopé et dont le dopage initial $N_{\mathrm{D}}-N_{\mathrm{A}}$ est uniforme et égal à $6,5 \times 10^{15} \mathrm{~cm}^{-3}$.

Après polissage mécanique et nettoyage chimique par une solution Br-méthanol suivi de rinçages, les contacts ohmiques sont effectués par dépôt d'étain et chauffage à $310^{\circ} \mathrm{C}$ sous atmosphère d'hydrogène en présence de $\mathrm{NH}_{4} \mathrm{Cl}$. Après nettoyage chimique, afin d'avoir une surface propre standard, l'échantillon 
est conservé en atmosphère normale pendant $24 \mathrm{~h}$ pour laisser se former une couche d'oxyde de quelques $10 \AA$. On effectue ensuite une vaporisation sous vide d'une couche d'Au de $400 \AA$ environ. Le contact Schottky obtenu est ainsi optimisé pour les mesures capacitives. La surface des diodes est de $0,8 \mathrm{~mm}^{2}$. Le courant de fuite en polarisation inverse à température ambiante est généralement de $1 \mathrm{~mA} / \mathrm{cm}^{2}$.V. Un spectre DLTS (Deep Level Transient Spectroscopy) est effectué avant irradiation pour mesurer la concentration de défauts éventuels. Le seul défaut résiduel est celui attribué à la présence du fer $\left(E_{\mathrm{C}}-E_{\mathrm{T}}=0,63 \mathrm{eV} \sigma=\right.$ $\left.300 \AA^{2}\right)$ en concentration très faible $\left(10^{11}\right.$ à $\left.10^{13} / \mathrm{cm}^{3}\right)$. Les irradiations sont faites au moyen d'un accélérateur à protons $150 \mathrm{keV}$ [7]. La tension d'accélération utilisée est de $100 \mathrm{kV}$. Les mesures sont effectuées in situ. Des expériences préliminaires nous ont permis de déterminer la dose d'irradiation compatible avec les mesures de profils de piège par double DLTS (DDLTS). Celle-ci se situe vers $10^{11} \mathrm{H}^{+} / \mathrm{cm}^{2}$. L'incertitude sur la dose reçue par l'échantillon est estimée à $20 \%$. On remarque que cette dose est, à peu près, la même que celle utilisée pour une étude similaire sur GaAs [3]. Le taux de compensation est sensiblement identique alors que la concentration en centres mesurés par DLTS est environ 100 fois plus faible dans InP que dans GaAs. Les irradiations aux électrons donnent un résultat identique [8].

\section{Résultats expérimentaux.}

3.1 IRRADIATION BASSE TEMPÉRATURE. - Les irradiations à $4 \mathrm{~K}$ et $77 \mathrm{~K}$ donnent des résultats strictement identiques : mêmes centres formés avec les mêmes concentrations. Aucun niveau n'apparaît par DLTS entre $4 \mathrm{~K}$ et $77 \mathrm{~K}$. On observe, par DLTS (Fig. 1) 4 pièges principaux nommés $A_{1}, A_{2}, A_{3}, A_{4}$ par ordre d'apparition dont les signatures, nombre créé par $\mathrm{H}^{+}$ $(100 \mathrm{keV})$ et domaine de stabilité sont donnés dans le tableau I. $A_{1}$ disparaît après chauffage à $100 \mathrm{~K}$ et $\mathrm{A}_{2}$ après chauffage à $160 \mathrm{~K}$. Ces résultats sont tout à fait comparables à ceux obtenus par irradiation aux électrons à $20 \mathrm{~K}$ [8]. Dans le domaine de température $77-160 \mathrm{~K}$, après recuits de $A_{1}$ et $A_{2}$, apparaissent plusieurs centres en concentration beaucoup plus faible. Il n'est pas possible de dire, à ce niveau, si $A_{3}$ et $\mathrm{A}_{4}$ ont été formés à partir de la disparition de $\mathrm{A}_{1}, \mathrm{~A}_{2}$ ou formés en même temps au cours de l'irradiation. Nous avons effectué une étude de profils de centres par mesure DDLTS en utilisant une procédure décrite antérieurement [3].

$A_{1}$ et $A_{2}$ : ces deux centres ont des profils gaussiens avec $R_{\mathrm{p}}=0,58 \mu$ et $\Delta R_{\mathrm{p}}=0,13 \mu$ (Fig. 2).

La valeur de $R_{\mathrm{P}}$ est celle mesurée par analyse SIMS pour des protons de $100 \mathrm{keV}$ par Mathiot et al. [9], le $\Delta R_{\mathrm{p}}\left(\mathrm{A}_{1}, \mathrm{~A}_{2}\right)$ est plus élevé que celui donné par ces auteurs $\left(0,05 \mu\right.$ pour profil $\left.\mathrm{H}^{+}\right)$. Ceci est dû, d'une part, au fait que la résolution spatiale maximum de nos mesures est donnée par la longueur de Debye $(0,05 \mu$ à $100 \mathrm{~K}$ et $0,07 \mu$ à $200 \mathrm{~K}$ ) et, d'autre part, $\Delta R_{\mathrm{p}}=$

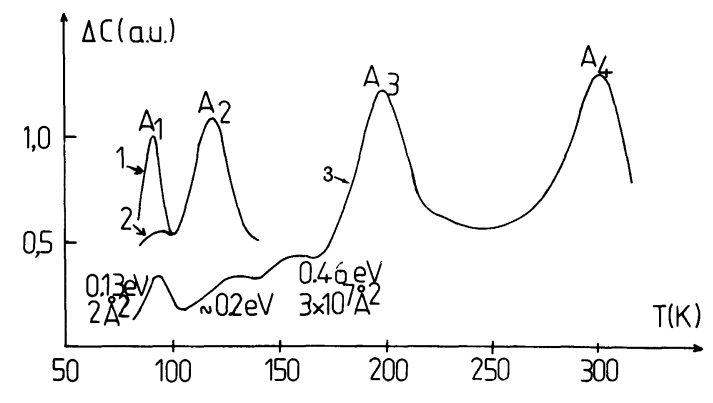

Fig. 1. - Spectre DLTS $e_{\mathrm{n}}=25,6 \mathrm{~s}^{-1}$ IRR $\mathrm{H}^{+} 100 \mathrm{keV}$, $5 \times 10^{11} \mathrm{~cm}^{-2} ; 1$. DLTS $77-140 \mathrm{~K}$ après irradiation à $77 \mathrm{~K} ; 2$. Après première montée en température à $140 \mathrm{~K}$; 3. Après première montée en température à $300 \mathrm{~K}, \operatorname{InP}: \mathrm{n}$ LEC MR $N_{\mathrm{D}^{-}} N_{\mathrm{A}}=6,5 \times 10^{15} \mathrm{~cm}^{-3}$.

[DLTS spectra $e_{\mathrm{n}}=25.6 \mathrm{~s}^{-1} 100 \mathrm{keV} \mathrm{H^{+ }}$ IRR : $5 \times$ $10^{11} \mathrm{~cm}^{-2} ; 1$. DLTS $77-140 \mathrm{~K}$ after irradiation at $77 \mathrm{~K}$; 2. After first annealing to $140 \mathrm{~K} ; 3$. After first annealing to $300 \mathrm{~K}$, InP : n LEC MR $N_{\mathrm{D}}-N_{\mathrm{A}}=6.5 \times 10^{15} \mathrm{~cm}^{-3}$.]

Tableau I. - Principaux centres créés par irradiation aux protons à $77 \mathrm{~K}$ sur $\mathrm{InP}$ massif $\mathrm{n}\left(6,5 \times 10^{15} \mathrm{~cm}^{-3}\right)$. $T$ : température $d u$ maximum du pic DLTS pour $e_{\mathrm{n}}=25,6 \mathrm{~s}^{-1} ; T_{\mathrm{r}}$ : température de recuit pour nos expériences; $N$ : nombre de défauts créés par proton de $100 \mathrm{keV}$;

[Main centres created at $77 \mathrm{~K}$ by proton irradiation on L.E.C. $\operatorname{InP}\left(\mathrm{N}_{\mathrm{D}}-\mathrm{N}_{\mathrm{A}}: 6.5 \times 10^{15} \mathrm{~cm}^{-3}\right) . T$ : temperature of the DLTS peak maximum for $e_{\mathrm{n}}=25.6 \mathrm{~s}^{-1}$; $T_{\mathrm{r}}$ : annealing temperature; $N$ : number of created defects per $100 \mathrm{keV}$ proton.]

\begin{tabular}{lcccc}
\hline & $\mathrm{A}_{1}$ & $\mathrm{~A}_{2}$ & $\mathrm{~A}_{3}$ & $\mathrm{~A}_{4}$ \\
\hline $\begin{array}{lcccc}E_{\mathrm{C}}-E_{\mathrm{T}}(\mathrm{eV}) \\
\sigma_{\mathrm{n}}\left(\AA^{2}\right)\end{array}$ & 0,16 & 0,22 & 0,36 & $\sim 0,7$ \\
\hline$T$ & - & 300 & 30 & - \\
\hline$T_{\mathrm{r}}$ & $90 \mathrm{~K}$ & $118 \mathrm{~K}$ & $195 \mathrm{~K}$ & $300 \mathrm{~K}$ \\
\hline$N$ & $100 \mathrm{~K}$ & $160 \mathrm{~K}$ & $375 \mathrm{~K}$ & $370 \mathrm{~K}$ \\
\hline Création directe & 0,25 & 0,1 & 0,3 & - \\
\hline Nomencl. $\left(^{*}\right)$ & $\mathrm{E}_{4}$ & $\mathrm{E}_{5}$ & $\mathrm{E}_{8}$ & $\mathrm{E}_{11}$ \\
\hline$v \mathrm{~cm}^{-1}$ & 0,0044 & 0,0053 & 0,028 & $\sim 0,33$ \\
\hline
\end{tabular}

(*) Comparaison avec les centres créés par irradiation électronique à $20 \mathrm{~K}$ [8]; $v$ : taux d'introduction des défauts.

[Comparison with $20 \mathrm{~K}$ electron irradiation induced centres [8]. $v$ : defect introduction rate $\left(\mathrm{cm}^{-1}\right)$.]

$0,05 \mu$ est une valeur calculée à partir d'un profil considéré comme gaussien alors que celui mesuré par SIMS est sensiblement plus large.

Le profil de $A_{3}$ est, à peu près, plat (Fig. 3). La mesure a été effectuée de deux façons : soit, après irradiation, 


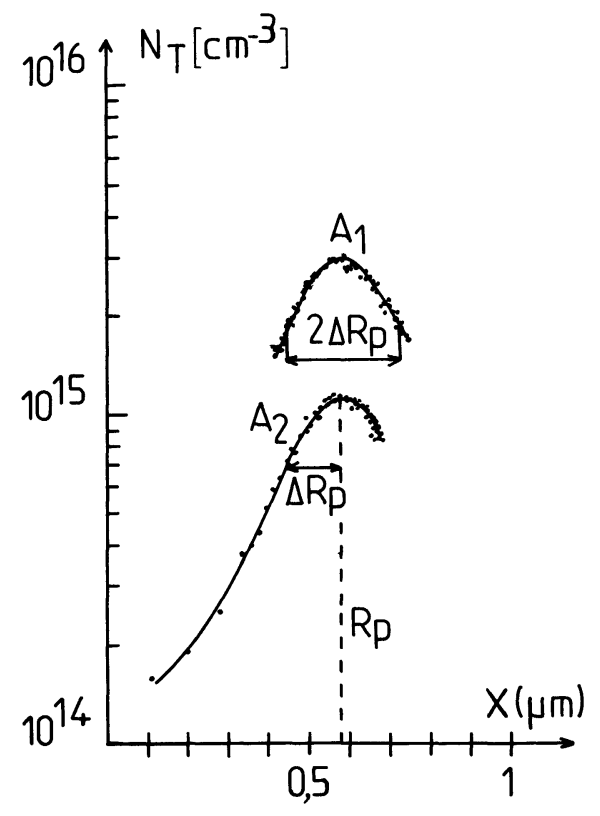

Fig. 2. - Profils de concentration des centres $A_{1}$ et $A_{2}$ déterminés par DDLTS (voir texte), $T=90 \mathrm{~K}\left(\mathrm{~A}_{1}\right)$, $T=115 \mathrm{~K}\left(\mathrm{~A}_{2}\right)$.

[Concentration profiles determined by DDLTS (see text), $\mathrm{A}_{1}(T=90 \mathrm{~K}), \mathrm{A}_{2}(T=115 \mathrm{~K})$.]

lors de son apparition à la première montée en température (procédure utilisée pour les profils de $A_{1}$ et $A_{2}$ ), soit après chauffage jusqu'à température ambiante et redescente à $200 \mathrm{~K}$. Les concentrations sont les mêmes dans les deux cas, $\mathrm{A}_{3}$ étant stable jusqu'à $350 \mathrm{~K}$ [8].

Le profil de $A_{4}$ n'a pu être mesuré, le courant de fuite à $300 \mathrm{~K}$ étant incompatible avec la précision demandée par la technique DDLTS (impossibilité de polariser suffisamment la diode). On observe que $\mathrm{A}_{4}$ est à peine stable à température ambiante (variation de $50 \%$ en 18 jours).

3.2 IrRADIATION À TEMPÉRATURE AMBIANTE. - Une irradiation à $300 \mathrm{~K}$ donne un résultat identique à une irradiation à $77 \mathrm{~K}$ à même dose et recuit à $300 \mathrm{~K}$.

\section{Conclusion.}

La quantité de résultats expérimentaux sur les défauts créés par irradiation dans InP est loin d'être aussi importante que pour GaAs. La majeure partie des défauts créés globalement en concentration iden-

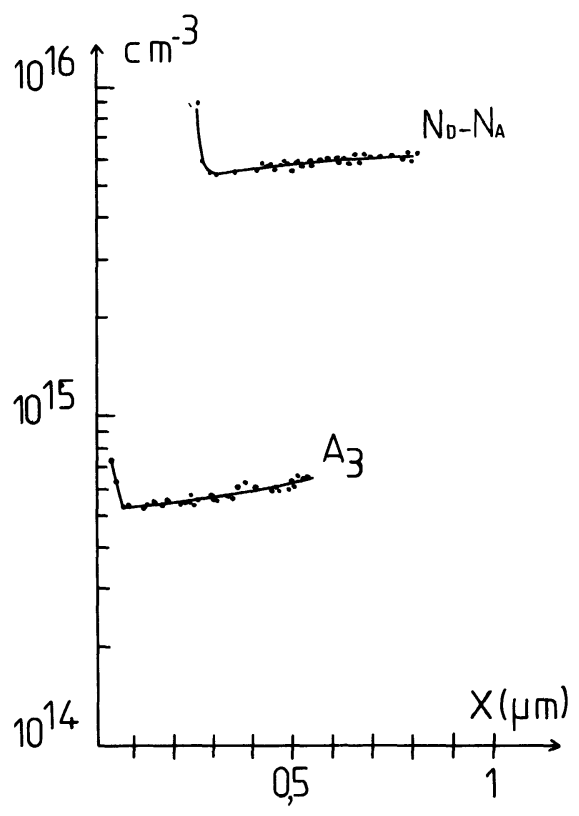

Fig. 3. - Profils de porteurs libres $N_{\mathrm{D}}-N_{\mathrm{A}}$ et profil de concentration du centre $A_{3}$ déterminé par DDLTS (voir texte). $T=205 \mathrm{~K}$.

[Free carrier concentration profile $N_{\mathrm{D}}-N_{\mathrm{A}}$, and $\mathrm{A}_{3}$ concentration profile determined by DDLTS (see text). $(T=205 \mathrm{~K})$.]

tique à ce que l'on trouve pour GaAs n'est pas observable par DLTS.

Nos expériences montrent :

- qu'une irradiation aux protons à basse température conduit à la création des mêmes types de défauts qu'après irradiation aux électrons,

- que le nombre de défauts créés par les protons de $100 \mathrm{keV}$ ne peut être corrélé aux taux d'introduction correspondants (rapports non constants). Ceci indique qu'il s'agit probablement de défauts complexes, conclusion déjà suggérée par le fait que les taux d'introduction sont faibles et diffèrent sensiblement dans la littérature $[5,8]$,

- qu'au moins l'un des deux centres principaux créés par irradiation à température ambiante $\left(\mathrm{A}_{3}\right)$ provient d'un processus de diffusion avant stabilisation.

Il apparaît vraisemblable que les défauts observés dans InP à température ambiante sont de nature plus complexe que dans GaAs. 


\section{Bibliographie}

[1] Donnelly, J. P., Hurwitz, C. E., Appl. Phys. Lett. $31 \quad$ [6] TAPster, P. R., Dean, P. J., Skolnick, M. S., J. Phys. C (1977) 418 . 15 (1982) L1007.

[2] Eirug Davies, D., Lorenzo, J. P., Deane, H. L., [7] Guillot, G., Nouailhat, A., Vincent, G., Baldy, M., Appl. Phys. Lett. 31 (1977) 256.

Chantre, A., Revue Phys. Appl. 15 (1980) 679.

[3] Loualiche, S., Nouailhat, A., Guillot, G., Solid. [8] Suski, J., Bourgoin, J. C., Sibille, A., 4e Conf. Internat. State Commun. 44 (1982) 41. « Lund " 1983 (à paraître).

[4] Brailovskit, E. Yu, Karapetyan, F. K., Tartachnik, [9] Mathiot, D., Huber, A. M., Perrocheau, J., Morillot, V. P., Sov. Phys. Semicond. 13 (1980) 1193.

G., Inst. Phys. Conf. ser. 56 (1981) 689.

[5] Levinson, M., Benton, J. L., Temkin, H., Kimerling,

L. C., Appl. Phys. Lett. 40 (1982) 990. 